\title{
Prevention is better than cure
}

\section{Attempts to reduce amyloid - $\beta$ in the brain have yet to show clinical benefits. Starting treatment early is the best hope, says Sam Gandy.}

$\mathrm{I}$ n 1907, Bavarian psychiatrist Alois Alzheimer published his description of a delusional woman who had slowly lost all cognitive function and died at 55 years of age. For decades thereafter, because of the patient's age, 'Alzheimer's presenile dementia' was considered a rare disease of mid-life. In the 1970s, neuropathologists realized that 'senile dementia' was indistinguishable from the disease described by Alzheimer ${ }^{1}$. The clinical picture of progressive brain dysfunction in association with the postmortem brain pathology of extracellular amyloid deposits ('plaques') and intraneuronal 'tangles' was renamed 'Alzheimer's disease', and its definition was broadened to include the major cause of dementia that we know today.

The accumulation of amyloid plaques is the key distinguishing feature of Alzheimer's disease, and research in the late 1980s identified the major plaque protein as the amyloid- $\beta$ peptide. In the 1990 s, scientists connected amyloid- $\beta$ pathology with genes encoding three proteins: the amyloid- $\beta$ precursor protein (APP), which is cleaved by $\gamma$-secretase to create amyloid- $\beta$, and two forms of presenilin (presenilin 1 and presenilin 2), which are involved in amyloid- $\beta$ generation" ${ }^{2}$ (see 'Finding risk factors, page S20). Together, mutations in these genes account for about 3\% of Alzheimer's disease, and insertion of one of these mutations into the mouse genome created the first transgenic mice to form amyloid plaques.

But what about the other $97 \%$ of Alzheimer's disease? Parsimony would dictate that amyloid- $\beta$ pathology might be central to all forms of Alzheimer's disease ${ }^{3}$. Less than five years after the plaque-forming mice were developed, the first amyloid- $\beta$ lowering drugs and vaccines were identified. However, initial human trials of the drugs were uninterpretable because they included no methods for measuring how much amyloid- $\beta$ is in the brain. That challenge was overcome in 2004 using positron emission tomography to identify amyloid plaques ${ }^{4}$.

And that's where the field stood for six years. Then, in 2010, researchers at the University of Turku in Finland showed that

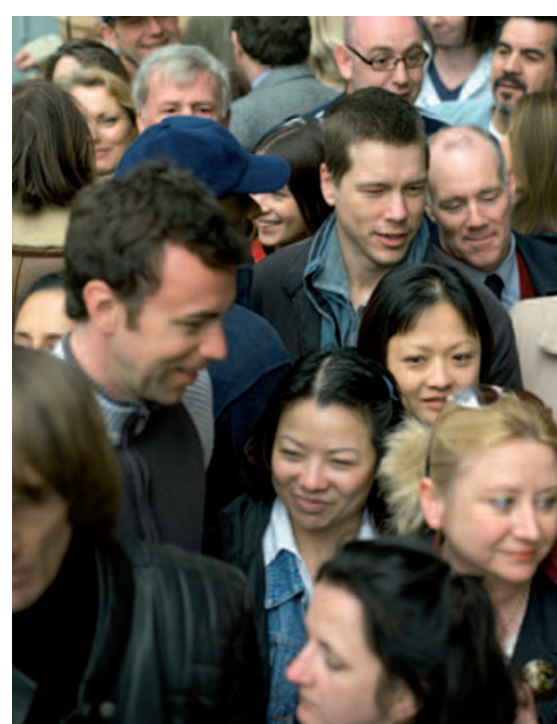

The process of Alzheimer's can begin 20 years before the mind shows signs of cognitive loss.

immunotherapy with an anti-amyloid- $\beta$ monoclonal antibody lowered plaque burden by around 25\%. Disappointingly, however, the treatment - an infusion of antibody every three weeks for 1.5 years brought no cognitive benefit for the patient ${ }^{5}$.

Why did this immunotherapy reduce brain plaques but fail to halt cognitive decline? Perhaps 1.5 years is not long enough or perhaps a $25 \%$ reduction in plaque burden is insufficient. To allow for this possibility, immunotherapy trials are continuing and results are expected in 2013. Another possibility is that the monoclonal antibody used might not recognize the most important neurotoxic conformation(s) of amyloid- $\beta$. Dozens of different monoclonal antibodies, as well as intravenous immunoglobulin, are now in clinical trials, in the hope that one or several will recognize and neutralize the most neurotoxic forms of amyloid- $\beta$.

There is at least one more possible interpretation of the Turku study: that therapy to lower amyloid- $\beta$ levels will never succeed in symptomatic patients. Brain imaging data from presymptomatic individuals who carry presenilin 1 mutations show that plaque accumulation starts $10-20$ years before clinical symptoms appear ${ }^{6}$. So if subjects enter trials at the first sign of cognitive impairment, they might already harbour substantial quantities of neurotoxic amyloid- $\beta$.

The best hope for therapies aimed at amyloid- $\beta$ levels, therefore, is to dose prophylactically to stop it building up in the first place. A diagnostic category of 'presymptomatic Alzheimer's disease' was recently proposed for subjects with strong biomarker-based evidence of disease but who are cognitively intact ${ }^{7}$. Nevertheless, in the absence of a perfect test for predicting who will develop Alzheimer's disease and when, prevention trials are highly daunting with regard to cohort size, trial duration and cost. The most obvious place to start is with carriers of presenilin 1, presenilin 2 or APP mutations, where disease risk and timing of onset are highly predictable. The Dominantly Inherited Alzheimer Network has been founded to identify carriers of pathogenic mutations worldwide and enrol them into prevention trials with amyloid- $\beta$-lowering agents.

The amyloid- $\beta$ odyssey of the past 25 years has shown that conquering Alzheimer's disease is not a matter of removing amyloid- $\beta$ plaques from the brain post hoc. But the role of amyloid- $\beta$ must be resolved, and our quest for effective interventions must be seen through to a successful end. Alzheimer's disease is already a problem for the healthcare systems of Western countries and is a growing threat to developing nations.

The best argument for sticking with strategies to lower amyloid- $\beta$ levels is that safe, effective compounds are within reach. Perfecting the selection of subjects and the timing of intervention could delay the onset of Alzheimer's disease substantially. A century of effort has brought us to a rational model for how Alzheimer's disease might begin, and we should not be discouraged by the prospect of another decade or two of work to settle the amyloid- $\beta$ issue and ultimately, we hope, defeat the illness. Prophylactic intervention to lower amyloid- $\beta$ levels is now the best hope.

Sam Gandy is neurology and psychiatry professor and associate director of the Alzheimer's Disease Research Center at Mount Sinai School of Medicine and the James J. Peters VA Medical Center in New York. e-mail:samuel.gandy@mssm.edu

1. Terry, R. D. \& Katzman, R. Ann. Neurol. 14, 497-506 (1983)

2. Bertram, L., Lill, C. M. \& Tanzi, R. E. Neuron 68, 270-281 (2010).

3. Gandy, S. J. Clin. Invest. 115, 1121-1129 (2005)

4. Klunk, W. E. et al. Ann. Neurol. 55, 306-319 (2004)

5. Rinne, J. O. et al. Lancet Neurol. 9, 363-372 (2010)

6. Knight, W. D. et al. Brain 134, 293-300 (2011).

7. Sperling, R. A. et al. Alzheimers Dement. 7 , 280-292 (2011)

The author declares competing financial interests: go.nature.com/hdiuds 